\title{
Aspectos sociais e biológicos da autoestima na gravidez e a assistência de enfermagem: revisão narrativa
}

\author{
Social and biological aspects of self-esteem in pregnancy and nursing care: narrative \\ review
}

Aspectos sociales y biológicos de la autoestima en el embarazo y atención de enfermería: revisión narrativa

Geovanna Lopes Dias¹, Camille Borges da Silva ${ }^{1}$, Erlisson de Sousa Araújoํㅜ, Bárbara Batista Lima$^{1}$, Adriana Paiva Camargo Saraiva ${ }^{1 *}$, Jerônimo Cavalcante Dantas Silva1, Raphael do Nascimento Gentil ${ }^{1}$, Thamyris Andrade de Oliveira, Priscilla Rodrigues Caminha Carneiro ${ }^{1}$.

\section{RESUMO}

Objetivo: Compreender os fatores que influenciam negativamente a autoestima de gestantes e como a assistência de Enfermagem pode atuar durante o pré-natal, sob aspectos sociais e biológicos. Revisão bibliográfica: Os artigos utilizados foram consultados nas bases de dados Scientific Eletronic Library Online (Scielo), Google acadêmico e Biblioteca Virtual em Saúde (BVS) por meio de palavras-chave combinadas entre si com o operador booleano AND, na busca por estudos científicos que abordassem problemas e soluções relacionados a baixa autoestima durante a gravidez. A revisão foi dividida em mudanças anatômicas e fisiológicas, alterações dermatológicas, fatores socioeconômicos e outros. De acordo com a literatura analisada, a baixa autoestima entre gestantes pode estar associada a estrias, celulite, modificações hormonais e físicas, bem como planejamento da gravidez, apoio familiar e do parceiro. Os meios relativos de solução como: o uso de hidratantes, protetor solar, o pré-natal de qualidade e o apoio familiar contribuem para melhorar a autoestima da gestante. Considerações finais: Pode-se dizer que a assistência de enfermagem durante o pré-natal é fundamental para fornecer informações de qualidade quanto às mudanças anatômicas e fisiológicas, e incentivar o parceiro e familiares ao apoio da gestante.

Palavras-chave: Autoestima, Enfermagem, Gravidez.

\begin{abstract}
Objective: To understand the factors that negatively influence the self-esteem of pregnant women and how nursing care can act during prenatal care, under social and biological aspects. Bibliographic review: The articles used were consulted in the Scientific Eletronic Library Online (SciELO), Google Scholar and Virtual Health Library (VHL) databases using keywords combined with the Boolean operator AND, in the search for scientific studies that addressed problems and solutions related to low self-esteem during pregnancy. The review was divided into anatomical and physiological changes, dermatological changes, socioeconomic factors and others. According to the analyzed literature, low self-esteem among pregnant women may be associated with stretch marks, cellulite, hormonal and physical changes, as well as pregnancy planning, family and partner support. Relative means of solution such as: the use of moisturizers, sunscreen, quality prenatal care and family support contribute to improving the pregnant woman's self-esteem. Final considerations: It can be said that nursing care during prenatal care is essential to provide quality information regarding anatomical and physiological changes, and to encourage the partner and family members to support the pregnant woman.
\end{abstract}

Key words: Self-esteem, Nursing, Pregnancy.

\footnotetext{
1 Universidade do Estado do Pará (UEPA), Conceição do Araguaia - PA.

*E-mail: adriana.saraiva@uepa.br
} 


\section{RESUMEN}

Objetivo: Comprender los factores que influyen negativamente en la autoestima de la gestante y cómo pueden actuar los cuidados de enfermería durante el prenatal, en aspectos sociales y biológicos. Revisión bibliográfica: Los artículos utilizados fueron consultados en las bases de datos Scientific Eletronic Library Online (SciELO), Google Scholar y Virtual Health Library (BVS) utilizando palabras clave combinadas con el operador booleano AND, en la búsqueda de estudios científicos que abordaran problemas y soluciones relacionados con baja autoestima durante el embarazo. La revisión se dividió en cambios anatómicos y fisiológicos, cambios dermatológicos, factores socioeconómicos y otros. Según la literatura analizada, la baja autoestima entre las embarazadas puede estar asociada a estrías, celulitis, cambios hormonales y físicos, así como a la planificación del embarazo, apoyo familiar y de pareja. Medios de solución relativos como: el uso de cremas hidratantes, protector solar, atención prenatal de calidad y apoyo familiar contribuyen a mejorar la autoestima de la gestante. Consideraciones finales: Se puede decir que el cuidado de enfermería durante el prenatal es fundamental para brindar información de calidad sobre los cambios anatómicos y fisiológicos, y para incentivar a la pareja y familiares a apoyar a la gestante.

Palabras clave: Autoestima, Enfermería, Embarazo.

\section{INTRODUÇÃO}

A autoestima é um autoconceito ou autopercepção que reflete um sentimento individual, que se baseia na forma pela qual o indivíduo se autoavalia positiva ou negativamente pelos seus atos, pensamentos e referências estéticas. A alta autoestima está associada a um afeto mais positivo e pode ser importante no enfrentamento de situações estressantes e emoções negativas (OPHEIN R, et al., 2020).

Quando se trata do período gestacional, as dificuldades na compreensão das transformações no corpo, a preocupação de suficiência em frente a si própria ou até pelo meio social inserido, podem impactar a aceitação de si mesma. Além de todas as mudanças físicas, a função do seu papel diante do feto dá lugar a dúvidas, inseguranças e certas limitações transformando a vida e a autoestima da mãe. Para manter a estabilidade dessa autoestima que oportuniza o indivíduo a socializar, a manter um ritmo de eficiência na execução das tarefas do cotidiano; isso também otimiza a forma pela qual o indivíduo tem a capacitação de superar as complicações e obstáculos (SEIBEL BL, et al., 2016).

A cada período gestacional, um novo desafio. Durante o primeiro trimestre, manifestam-se os primeiros sintomas e mudanças corporais, que interferem na percepção da grávida, gerando desconfortos e angústias que podem causar um declínio da autoestima e dificuldade de aceitação. No segundo trimestre, as metamorfoses corporais ficam mais perceptíveis e marcam o início da relação mãe-filho e sociedade, intensificando a construção da relação com o feto. No terceiro trimestre, impasses psicológicos se acentuam com uma nova fase de adaptações fisiológicas e anatômicas, sociais e questões ligadas ao parto e puerpério. Outrossim, é a exclusão dos padrões estéticos sociais o motivo a qual tornam a mulher insegura sobre seu corpo (URBANETZ AA, 2021).

Outrossim, a assistência da enfermagem durante a promoção da autoestima e saúde física da gestante, precisa ser de forma humanizada e integral, pois a partir daí será possível a identificação do diagnóstico de acordo com a NANDA. Ademais, o pré-natal é um momento em que há a preparação da mãe nos cuidados para o parto, principalmente às questões físicas. Entretanto, faz-se necessário a abordagem de preocupação com o psicológico da gestante para compreender as alterações negativas em sua forma completa e prestar o apoio emocional necessário (TOMASCHEWSKI-BARLEM JG, et al., 2016).

Desta forma, o presente artigo tem como objetivo compreender os fatores que influenciam negativamente a autoestima de gestantes e como a assistência de Enfermagem pode atuar durante o prénatal, sob aspectos sociais e biológicos.

\section{REVISÃO BIBLIOGRÁFICA}

Optou-se alcançar os objetivos por meio da revisão narrativa da literatura. Os artigos utilizados foram consultados nas bases de dados Scientific Eletronic Library Online (Scielo), Google acadêmico e Biblioteca 
Virtual em Saúde (BVS) por meio de palavras-chave combinadas entre si com o operador booleano AND, na busca por estudos científicos que abordassem problemas e soluções relacionados a baixa autoestima durante a gravidez. A revisão foi dividida em mudanças anatômicas e fisiológicas, alterações dermatológicas, fatores socioeconômicos e outros.

\section{Mudanças anatômicas e fisiológicas}

Os ajustes posturais podem ser percebidos ao decorrer da gestação, como o aumento do peso, tamanho das mamas e crescimento do útero (anteriorizado dentro da cavidade abdominal), esses fatores são responsáveis por gerar diversos desconfortos na gestante, seja pela qualidade de vida ou pelo significado desses ajustes diante a sua percepção de belo (CONTI MHS, et al., 2003).

Outro fator que deve ser descrito é o inchaço provocado nas articulações. O edema de membros inferiores é um dos sinais mais comuns neste período, destacando-se como um dos mais desconfortáveis para as mulheres afetadas, pois, com frequência, associa-se a sintomas como dor, cansaço, sensação de peso e parestesia nos pés e pernas acometidos, além do componente de estético que tanto incomoda as mulheres (BAMIGBOYE AA e HOFMEYR GJ, 2006).

No primeiro trimestre da gestação (1 $1^{\mathrm{a}}$ a $12^{\mathrm{a}}$ semana), ocorrem algumas alterações fisiológicas, em que estas marcam o começo do aumento do peso corporal (MEIRELES JFF, et al., 2016). Como também ocorrem mudanças no humor das gestantes, que se apresenta já bem frequente nessa fase inicial da gestação, cujas mudanças estão intimamente ligadas com as alterações do metabolismo, onde há a elevação da concentração dos hormônios da mulher, o estrogênio e a progesterona (BURTI JS, et al., 2006).

Durante a gestação, a progesterona inibe a musculatura uterina, permitindo então que não ocorra a expulsão do feto; ela também promove a redução dos tônus de músculos lisos, em que pode ocorrer diminuição do peristaltismo, náuseas, constipação, dilatação de veias, estase de urina, diminuição da pressão diastólica e redução da tensão na pressão intra-alveolar e arterial (podendo causar hiperventilação) (BURTI JS, et al., 2006).

Já no segundo trimestre (entre a $13^{\text {a }}$ a $25^{\underline{a}}$ semana), o aparecimento e crescimento da barriga acontece, logo é possível observar com mais nitidez as mudanças corporais (MEIRELES JFF, et al., 2016). Em que os órgãos já estão mais comprimidos devido ao constante crescimento do bebê no útero, um exemplo a ser citado é a bexiga, que vai se comprimido conforme o crescimento do bebê, e a gestante passa a ter mais vontade de ir ao banheiro durante o dia.

E no terceiro e último trimestre (da 26 $6^{a}$ semana em diante), é o período em que há um aumento de queixas físicas por parte da gestante, pois é o momento em que o desenvolvimento do bebê é maior e por consequência um maior peso corporal da gestante (MEIRELES JFF, et al., 2016). Estas alterações fisiológicas e anatômicas que acontecem durante a gestação pode ser um fator que afeta diretamente a autoestima das gestantes, em relação a como ela se ver diante de tais mudanças que acontece em seu próprio corpo.

\section{Alterações dermatológicas e citológicas}

Durante a gravidez e o pré-natal a grande preocupação se refere a saúde do feto e da mãe. Entretanto, na realidade pode ocorrer obstáculos notáveis através de fatos inesperados, como anomalias com o feto ou uma gravidez de risco. Ao se trata de meiose e período embriológico, é notado que os erros dessas multiplicações e divisões celulares que possibilita a descoberta de uma anomalia no feto, o que agride diretamente a autoestima da mãe, isso porque o enfrentamento dessa situação pode desestabilizar a sua saúde psicológica e agravar a gestação, indiciando o alto nível de estresse e tensões se acentuam, seja pelo sentimento de insegurança, ou pela sensação de insuficiência e frustração. Esse incidente decorre pelo motivo de que as mães, em sua maioria, idealizam a gravidez e o bebê sem complicação de saúde, a partir disso é gerado os primeiros pensamentos da visão da gestante sobre ela, diante a capacidade de autocuidado e atenção (VINCENTE SECR, et al., 2016). 
Como ainda, outra aflição de algumas gestantes é ser portadora de uma infecção sexualmente transmissível como: a sífilis, herpes, Imunodeficiência Humana (HIV/AIDS). Decerto, que na gestação humana o feto encontra-se envolvido pela placenta, mas, mesmo assim, existe uma preocupação maternal com a troca de substâncias durante o parto e a formação uterina, tornando a mãe insegura por não ter controle dessa situação, diminuindo assim sua autoestima e a qualidade de vida.

Destaca-se, que em questão das células sanguíneas ocorre as mudanças hematológicas, onde os níveis de ferro que asseguram o desenvolvimento do feto e a produção de hemoglobina se afetam claramente caso ocorra uma baixa de nível, a qual possibilita o surgimento de patologias como a anemia, o que na gestante causa frustrações que podem induzir um quadro de gravidez de risco, além do mal-estar, a preocupação, e desestabilização da saúde física interferem na autoestima da mãe já que há a ausência da qualidade de vida, o que instiga a mãe ao isolamento, limitações nas atividades de rotina e tensões frequentes (SOUZA Al, et al., 2002).

Vale ressaltar, sobre as mudanças dermatológicas durante a gravidez, sendo essas: as estrias, as celulites e os melasmas. Como pode-se ver, uma das transformações que ocorre no tecido epitelial e no tecido conjuntivo são as estrias, caracterizadas por serem uma atrofia tegumentar adquirida (URBANETZ $A A, 2015)$.

As estrias, decorrem de rupturas das fibras elásticas que incidem como lesões lineares e paralelas e seguem as linhas de clivagem do tecido, se iniciam com aspecto avermelhado e posteriormente esbranquiçadas e brilhosas. Ainda, não se tem uma explicação específicas para o surgimento de estrias, porém, existem teorias para esclarecer a aparição dessas ranhuras na pele (URBANETZ AA, 2021).

Outrossim, a fundamentação das estrias também está relacionada com a gestação, pois, a teoria endócrina defende que a manifestação de estrias é relativa à alteração de hormônios, especificamente os corticoides. Em outro caso, a teoria infecciosa defende que os modos de infecções podem causar avaria às fibras elásticas, gerando as estrias. Por outro lado, a teoria mecânica é a teoria que defende o surgimento das estrias a partir da deposição excessiva de gordura no tecido adiposo, sendo assim, pode ocorrer o extravio das fibras elásticas dérmicas, uma exemplificação disso, são as mulheres no período de gestação (GUIRRO E e GUIRRO R, 2003).

Sendo assim, é perceptível a influência dessa alteração na autoestima das gestantes, já que essa lesão é considerada irreversível, tendo apenas amenizações diante procedimentos estéticos. Outrossim, sabe-se que na contemporaneidade é estipulado um padrão do que é belo, o que atinge diretamente as gestantes principalmente as que têm peso excessivo e gestação de mais de um feto, nesses casos é acentuado o surgimento de estrias pela sobrecarga elástica na pele.

Diante desse contexto, a mãe cultiva um modelo corporal antes do período gestacional, que é ressignificado devido a essas transformações inevitáveis das adaptações para o parto, através desse fator situacional é facilitado a ocorrência de estrias. Dessa maneira, essa alteração dermatológica corrobora não só no período gestacional, mas também no pós-parto, o que afeta a longo prazo o encadeamento do amor próprio da mulher.

Além dessa mudança na pele, existem as manchas que atingem diretamente às gestantes, essas manchas dermatológicas ou melasmas, são modificações na coloração da pele das gestantes que podem ser avermelhadas de castanho claro ao negro. Com isso, este fato ocorre devido a alteração nas populações de melanócitos, além da contribuição de progesterona e estrogênio, através da elevação dos níveis de Hormônio Melanócito Estimulante (MSH). Demais, esta pigmentação faz-se presente em $90 \%$ das pacientes grávidas, com presença nos mamilos; aréolas; axilas; genitais; períneo; anus; parte interna das coxas; no pescoço e na face (FERNANDES LB, et al., 2014).

O fibroedema gelóide é considerado uma das principais modificações das características da pele, que afeta em maior número as mulheres, causando problemas funcionais, por exemplo: emocionais e principalmente a perda da autoestima. A lipodistrofia ginóide (celulite) pode ser estipulada como uma patologia multifatorial, que sucede a degeneração do tecido adiposo, que faz passagem pela fase de 
alteração de matriz intersticial, estase microcirculatória e hipertrofia dos adiposos, juntamente com fibrose cicatricial (KEDE MPV e SABATOVICH O, 2004; FROZ MG e GIACOMOLLI C, 2014). Outrossim, pode ser um incômodo se olharmos pelo ponto de vista estético, esta enfermidade promove problemas de ordem psicossocial, ou seja, a exigência imposta pela sociedade sobre o estereótipo estéticos.

Percebe-se que a alteração da pele ocorre com diversas concavidades irregulares semelhantes à aparência de "casca de laranja" afetando especialmente as áreas dos glúteos e das coxas e com veemência em panturrilhas, braços e abdômen. Além disso, o fator agravante da celulite vem de hábitos inadequados, como má alimentação, sedentarismo, estresse, patologias, medicamentos e a gravidez. Diante disso, vê-se que o fator mais agravante é o que mais afeta as mulheres grávidas, isso por causa da aceleração no desequilíbrio hormonal (FROZ MG e GIACOMOLLI C, 2014).

\section{Fatores socioeconômicos}

A antropologia social possui enorme influência na autoestima da mulher durante o período gestacional. Assim, relações interpessoais e fatores socioeconômicos podem interferir diretamente na autoestima da gestante, sendo elas relacionadas à aceitação da família, união marital, faixa etária do período gestacional, nível de escolaridade e planejamento. Isso causa o abalo na saúde psicológica da grávida tornando-a tensa e preocupada, o que facilita a dispersão do cuidado a si e a gravidez (PEREIRA MFL, 2013).

A princípio, as gestantes que não possuem apoio familiar estarão propícias a diminuição da sua autoestima, sendo evidenciada pela sensação de incapacidade, medo de rejeição, tendência à procrastinação e dificuldade em aceitar as próprias limitações. Esse fardo fica mais evidente quando é colocada a situação socioeconômica, já que a falta de estrutura para um bom desenvolvimento da gestação é um fator no qual possibilita o desencadeamento de baixas perspectivas futuras e a redução da autoestima. Com certeza, o medo de morrer e não conseguir cuidar do bebê são alguns fatores que as gestantes carregam durante a gravidez (CORDEIRO EAO e SILVA LMS, 2018).

Quando se trata de felicidade, cada indivíduo possui suas metas e expectativas, que o encorajam a prosseguir e superar os obstáculos na vida. Assim, é importante salientar que os planejamentos de perspectivas futuras para o bebê podem influenciar diretamente no modo em que a gestante irá desenvolver essa gestação. Visto que, a gravidez é um dos momentos mais esperado por grande parte das mulheres, desse modo, a expectativa causada pode interferir na sua confiança e autoestima. Embora, em alguns fatores a gravidez seja encontrada como algo negativo, não pelo acontecimento do período gravídico, mas, pela aceitação da gestante, da cultura e a da sociedade em que se situa (FRANKL VE, 2008).

\section{Outros fatores}

A humanização no atendimento às gestantes é outro ponto muito relevante. Portanto, uma vez que elas se encontram com as mudanças biológicas interferindo ativamente no seu humor, ainda não se sabe bem as dificuldades acometidas no período gravídico, seja no âmbito psicológico, social ou econômico a maior preocupação do profissional de saúde é garantir o acolhimento e atendimento de qualidade para ajudar no processo vital (TOMASCHEWSKI-BARLEM JG, et al., 2016).

Outrossim, a enfermagem possui um papel importante na assistência do pré-natal e no acolhimento, através da função de prestar apoio emocional e educar sobre os assuntos desconhecidos e dúvidas das grávidas, promovendo saúde psicológica seja nas/com consultas, campanhas, palestras. Com isso, a gestante é acometida a ter posse de informações que a ajude a enfrentar as complicações com a autoestima, além de possui também o apoio emocional e científico do enfermeiro, the proporcionando vivenciar e repassar esse conhecimento afetando tanto a educação do parceiro ou/e da família (TOMASCHEWSKI-BARLEM JG, et al., 2016).

No momento em que a grávida entra em contato com a equipe de saúde, na unidade de saúde ou na comunidade, convém então a unidade de saúde compreender os múltiplos significados da gestação para aquela mulher e sua família. Logo, colocando em prática a Teoria Transcultural de Madeleine, em que esta teórica apresenta a metodologia, na qual o profissional de enfermagem ao realizar a interação com o 
paciente, em diversas situações de assistência, deve fazer a utilização de ações profissionais, de forma a preservar, negociar ou repadronizar os cuidados, por buscar então a congruência cultural (MONTICELLI M, et al., 2010).

\section{CONSIDERAÇÕES FINAIS}

De acordo com a literatura científica analisada, nota-se que a autoestima na gravidez é abalada por questões como o surgimento das estrias, celulites, edemas, anomalias no feto, influência do meio social, qualidade de vida, alterações hormonais e físicas. Pode-se dizer que a assistência de enfermagem durante o pré-natal é fundamental para fornecer informações de qualidade quanto às mudanças anatômicas e fisiológicas, e incentivar o parceiro e familiares ao apoio da gestante. Nesse caso, seria importante a ação da enfermagem na solução dos obstáculos de enaltecimento do amor-próprio nas gestantes seja pela assistência direta ou encaminhamento a outros profissionais de saúde. Ainda, sobre as hipóteses de solução, pode-se propor estratégias de incentivo ao autocuidado da gestante.

\section{REFERÊNCIAS}

1. BAMIGBOYE AA, HOFMEYR GJ. Interventions for leg edema and varicosities in pregnancy. What evidence? Eur $\mathrm{J}$ Obstet Gynecol Reprod Biol, 2006; 129(1):3 -8.

2. BURTI JS, et al. Adaptações fisiológicas do período gestacional. Fisioterapia Brasil, 2006; 7(5): 375-380.

3. CONTI MHS, et al. Desconfortos musculoesqueléticos da gestação - uma visão obstétrica e fisioterápica. Femina, 2003; 31(6): 531-535.

4. CORDEIRO EAO, SILVA LMS. Autoestima na gestação: Prevenção e Proteção Social. Revista Científica do Unisalesiano, 2018; 9(19): 427-439.

5. FERNANDES LB, et al. Alterações dermatológicas na gravidez: revisão da literatura. Femina, 2014; 42(2): $102-108$.

6. FRANKL VE. Em busca de sentido: um psicólogo no campo de concentração. Petrópolis: Vozes, 2008; 196p.

7. FROZ MG, GIACOMOLLI C. Celulite: Diferentes nomes para um só problema. UNICRUZ. Disponível em: https://document.onl/documents/celulite-diferentes-nomes-para-um-so-problema-celulite-sao-drenagemlinfatica.html. Acesso em: 24 nov. 2020.

8. GUIRRO E, GUIRRO R. Fisioterapia Dermato-Funcional: Fundamentos, recursos e patologias. 3 ed. São Paulo: Manole, 2003; 584p.

9. KEDE MPV, SABATOVICH O. Dermatologia Estética. São Paulo: Atheneu, 2004; 771p.

10. MEIRELES JFF, et al. Imagem corporal de gestantes: um estudo longitudinal. J Bras Psiquiatr, 2016; 65(3): 223230.

11. MONTICELLI M, et al. Aplicações da teoria transcultural na prática da enfermagem a partir de dissertações de mestrado. Texto e Contexto Enferm, 2010; 19(2): 220-228.

12. OPHEIM R, et al. Self-esteem in patients with inflammatory bowel disease. Qual Life Res, 2020; 29 (7):1839-1846.

13. PEREIRA MFL. Recursos Técnicos em Estética Volume I. São Caetano do Sul: Difusão editora, $2013 ; 1: 442$.

14. SEIBEL BL, et al. Psicologia positiva: teoria, pesquisa e intervenção. Curitiba: Ed. Juruá, 2016; 368p.

15. SOUZA Al, et al. Alterações hematológicas e gravidez. Revista brasileira de hematologia e hemoterapia, 2002; 24(1): 29-36.

16. TOMASCHEWSKI-BARLEM JG, et al. Promovendo a autoestima na gestação: foco no acolhimento. Revista Cofen, 2016; 7(2): 83-86.

17. URBANETZ AA. Ginecologia e Obstetrícia Febrasgo para o médico residente. ํo Ed. São Paulo: Manole, 2021; 1512p.

18. VICENTE SECR, et al. Estresse, ansiedade, depressão e coping materno na anomalia congênita. Estudos de Psicologia, 2016; 21(2): 104-116. 